SECTION 2

SAFETY STANDARDS

\& MANUFACTURING

\title{
Electrical safety in the use of biofeedback instruments
}

\author{
ALAN T. POPE \\ FDR Veterans Administration Health Care Facility, Montrose, New York 10548
}

\begin{abstract}
Electrical hazards associated with the use of medical apparatus are described with emphasis on biofeedback instruments. Safety standards specify safe current levels for instruments in normal operation; however, the user can take steps to provide back-up protection against hazards caused by component malfunction or breakdown. Measures for protection of patient and operator are outlined, including instrument design considerations and guidelines for the user to follow in order to provide a margin of safety.
\end{abstract}

As biofeedback devices are used in a wider range of applications and environments, it is important for the user to be aware of possible electrical hazards to the patient and to the operator. This awareness is especially advisable in situations where the biofeedback apparatus is connected to auxiliary, possibly linepowered, apparatus. The content of this article is taken from information compiled in the process of implementing a computer-augmented biofeedback system (Pope \& Gersten, 1977).

After considering the effects of electricity on the body, this paper will describe electrical hazards associated with the use of medical apparatus, including biofeedback instruments, and measures for protection against these hazards.

\section{EFFECTS OF ELECTRICAL CURRENT ON THE BODY}

For electrical current to flow through the body, a difference of potential (voltage source) must exist between at least two conductive contacts to the body. The intensity of electrical effect depends upon the magnitude of the current, which is directly related to the voltage between the contacts and inversely related to the resistance of the current path through the body (Ohm's law). The largest component of body resistance is normally skin resistance, which is inversely proportional to contact area. Dry intact skin has a resistance of about $15,000 \mathrm{ohm}$ to nearly $1 \mathrm{million} \mathrm{ohm} / \mathrm{cm}^{2}$ (Dalziel,

This work is supported by the Medical Research Service of the Veterans Administration. Requests for reprints should be addressed to Alan T. Pope, Psychology Service, FDR Veterans Administration Health Care Facility, Montrose, New York 10548.
1972; Pfeiffer, 1968). This high resistance provides a measure of natural protection against shock hazard. However, electrode paste or jelly can reduce this natural protection by electrolyte action and mechanical abrasion to as little as $2,500 \mathrm{ohm} / \mathrm{cm}^{2}$ (Pfeiffer, Note 1).

Since the heart is the organ most susceptible to electrical current, a patient with a direct connection from outside the body to the heart, such as with cardiac catheterization, is considered electrically susceptible. The effect of electrical current flowing between the heart contact and any other body contact is termed microshock (Weibell, 1974). Any time the normal skin resistance of a patient is in any way bypassed, the patient becomes electrically vulnerable to some degree. The effect of current between skin-surface contacts comes under the category of macroshock. Most present biofeedback applications involve only the possibility of a macroshock hazard.

\section{Range of Effects}

The effect of $60-\mathrm{Hz}$ ac on the body, through surface contact, ranges from the threshold of perception, through "let-go" current and ventricular fibrillation, to cardiac standstill. The diagram in Figure 1 is a composite drawn from several sources (Bernstein, 1975; Cromwell, Weibell, Pfeiffer, \& Usselman, 1973; Weibell, 1974; Pfeiffer, Note 1) and represents the widest ranges reported for thresholds of the various effects for current of $1 \mathrm{sec}$ or at least one heart cycle duration.

The threshold of perception at which a tingling sensation is felt varies with degree of contact and among individuals. This effect is not dangerous in itself but may cause secondary accidents due to the startle reaction (e.g., a fall from a ladder). Above the "let-go" current 


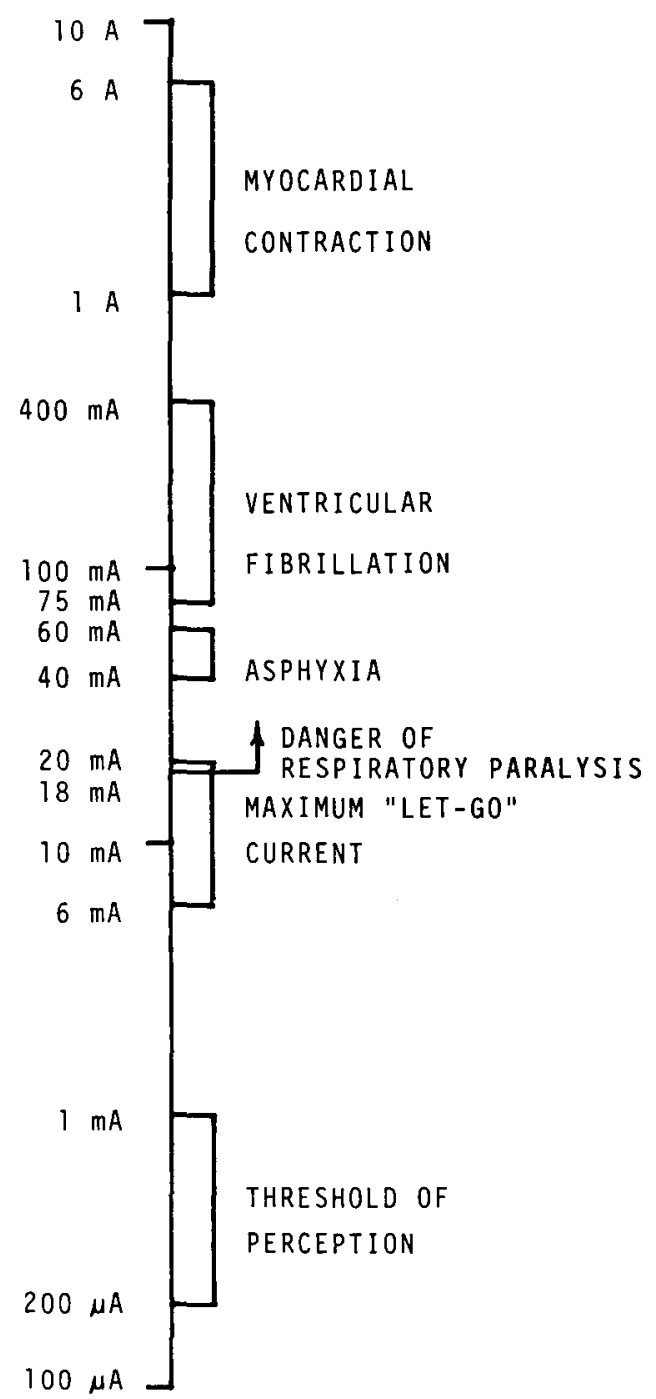

Figure 1. Thresholds of various effects of externally applied current on the body.

level, the victim cannot voluntarily release his grasp on the conductor and may be subjected to lethal current levels due to decreased body resistance (Bernstein, 1975).

When the current path includes the chest region, the effects on the heart and/or respiration can be life threatening. Respiratory paralysis can be caused by current affecting the respiratory control center in the medulla of the brainstem, in which case artificial respiration can help (Bernstein, 1975). Respiratory paralysis can also occur if the chest muscles are tetanized by an electrical current, resulting in suffocation, unless the electrical source is removed in time.

The most frequent cause of death in electrical accidents is ventricular fibrillation, an uncoordinated asynchronous contraction of the cardiac ventricular muscle fibers that defeats the heart's pumping action. Unconsciousness occurs in less than $10 \mathrm{sec}$ due to loss of blood circulation, and irreversible brain damage can occur in 4 to 5 min unless cardiopulmonary resuscitation is maintained until a defibrillator can restore the heart rhythm (Bernstein, 1975). The current necessary for fibrillation appears to be directly related to body weight and inversely related to the duration of the electrical shock. A measure of natural protection against fibrillation with surface contacts is provided by the distribution of current within the body that allows only a small part of the applied current to pass through the heart.

Strong electrical current can completely override the signals of the heart's natural pacemaker. The heart muscle contracts momentarily, then goes into fibrillation if the ac current persists.

\section{Safety Standards}

Electrical safety standards for medical instruments take into consideration the degree of electrical susceptibility due to degree and location of contact. Standards specify maximum limits on the current that may flow through the body as a result of the use of the apparatus. A substantial margin of safety is established between these limits and the dangerous current magnitudes described above.

The Safe Current Limits Standard for medical apparatus (SCL 12-78) of the Association for the Advancement of Medical Instrumentation (AAMI) has been adopted by the Executive Board of the Biofeedback Society of America and accepted by the National Association of Biofeedback Instrument Manufacturers. This standard is the only generic national standard for medical instrumentation that has been approved by the American National Standards Institute (ANSI). The AAMI standard sets risk-current limits for line- and batteryoperated apparatus when used singly or with auxiliary equipment. The standard specifies the test procedures for measurement of risk current.

Risk currents include leakage currents, surge currents, and measurement currents. Fault currents are excluded from the risk-current standards, and currents used for therapeutic purposes are not considered risk currents. Leakage currents are currents flowing between conductors that are intended to be insulated from each other. Surge currents are usually associated with tuming apparatus on or off. Measurement currents are intentionally passed through the body to measure, for example, skin conductance or electrode contact impedance. A fault current flows as the result of an accidental connection (short) between conductors, when at least one is energized. Under certain conditions, a fault current can flow due to a conductor break.

\section{HAZARD CONDITIONS}

Pfeiffer (Note 1) has developed a generalized model of the electrical accident (Figure 2) that is useful in describing hazard conditions and methods of protection 


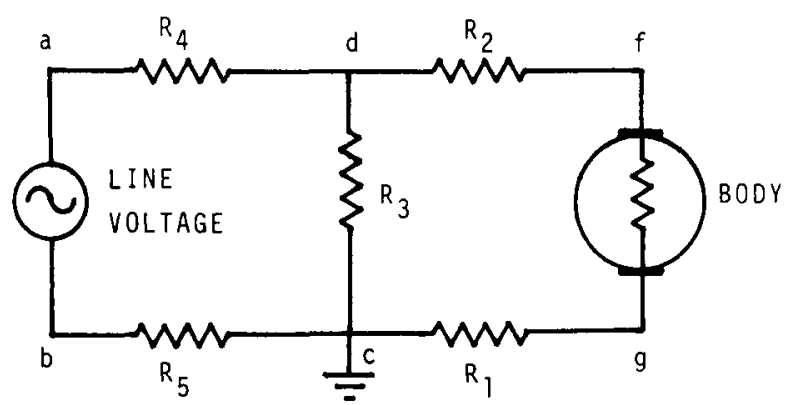

Figure 2. Generalized model of electrical accidents (Pfeiffer, Note 1).

against them. The locations of the resistances represented in Figure 2 for an equipment configuration example are shown in Figure 3.

The equipment leakage resistance $\left(\mathrm{R}_{\mathbf{4}}\right)$, through which leakage current flows, is actually an impedance, since it usually includes both a resistive and a reactive component. Capacitive coupling between the power transformer primary and core and case, between lineconnected parts and equipment case, between the hot wire and ground in long power cables, and in radiofrequency (RF) line filters contributes to the reactive component. Resistive leakage paths can develop in devices such as motors, in which dust and moisture collect between conductors. Leakage-current magnitude is rarely sufficient to pose a macroshock hazard.

\section{Fault Hazards}

A macroshock hazard is created by an accidental connection between an energized component and a metal equipment case (i.e., when the equipment leakage resistance, $\mathbf{R}_{\mathbf{4}}$, becomes essentially zero). This hazard condition can be particularly dangerous if the equipment grounding resistance, $R_{3}$, is large due to a poor or broken case-to-power-system ground connection (Figure 2). Three points along this ground path are particularly vulnerable: the ac cable and plug, the outlet ground connection, and the receptacle-to-power-system ground connection, especially when this last link is provided by conduit pipe. Conduit is a notoriously poor conductor, due primarily to corroded or loose connections. A temporary hazard can exist when a spilled conductive fluid (e.g., a salt-containing solution) accidentally shorts a conductor to the case.

In some configurations, the patient may be deliberately grounded (Point $g_{1}$, Figure 3 ) or have access to grounded metal objects (Point $\mathrm{g}_{2}$, Figure 3). A grounded patient (patient grounding resistance, $R_{1}$, essentially zero) is vulnerable in the event of a line-to-case short ( $R_{4}$ essentially zero). When input common is connected to the equipment case (patient lead resistance, $R_{\mathbf{2}}$, essentially zero), the above conditions place the patient directly across line voltage (Figure 2).

The equipment ground connection is intended to cause the installation's circuit breaker or fuse for the hot conductor to trip in the event of a ground fault (i.e., when both $R_{3}$ and $R_{4}$ are close to zero). However, the response time of a breaker is such that a dangerous voltage level could exist across even a small equipment ground resistance $\left(R_{3}\right)$ for a sufficient time to pose a hazard to both patient and operator.

\section{AAMI Specifications}

The AAMI Safe Current Limits Standard specifies the maximum risk current allowed to flow under specified test conditions through a standard test load from patient connections (Points f, Figures 2 and 3) to both exposed metal (Point d) and power ground (Point c). For apparatus with surface contacts, the limit is set at 50 microA. Risk current for isolated patient connection (electrically susceptible) is restricted to 10 microA at the apparatus terminals and 20 micro $A$ at the patient end of a patient cable. Risk current in the grounding conductor of cordconnected apparatus (Points c-d, Figures 2 and 3 ) is limited to 100 microA.

\section{METHODS OF PROTECTION}

Good instrument design and construction practices, such as suitable insulation and safe conductor spacing, can provide devices that meet the requirements of the standard for surface contact applications. However, there are protective measures that can aid in compliance with the most stringent standards and also protect against fault-current hazards as well. There are also precautions the user can take to provide an additional margin of

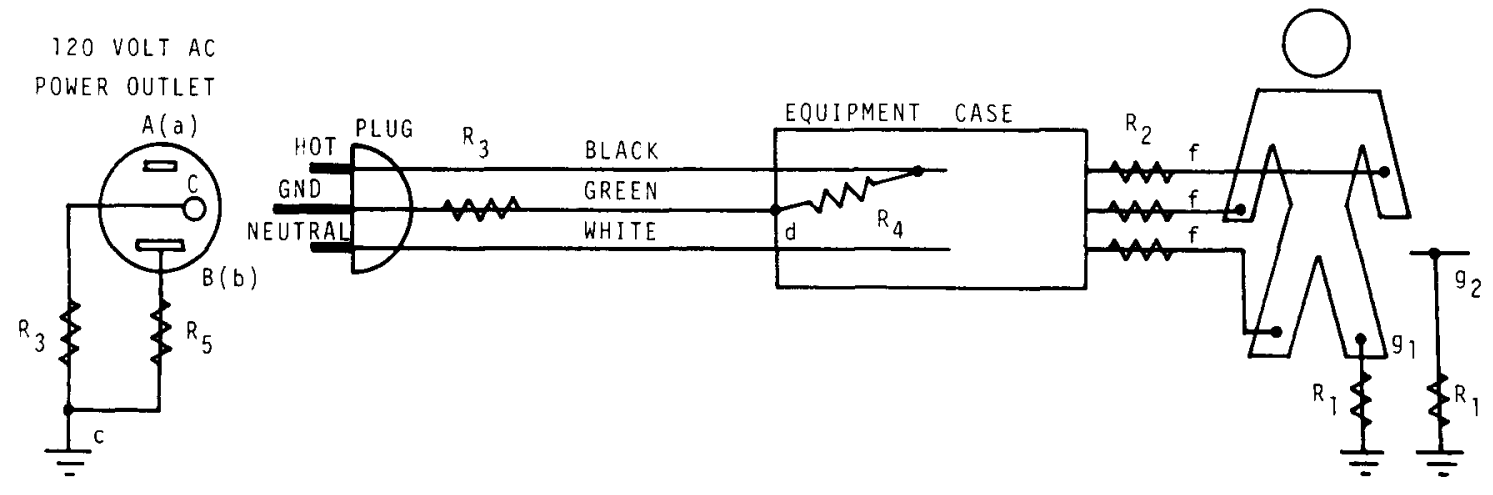

Figure 3. Example of equipment configuration. 
safety. When connecting battery-powered biofeedback instruments with line-powered equipment, the user should follow the manufacturer's precautions concerning interconnection.

Electrical safety measures are basically intended to isolate the patient and to prevent electrical potential differences between conductive objects in the patient environment. Redundant measures provide back-up protection. To keep the current through the body at a safe level, one or more of the resistances in Figures 2 and 3 may be manipulated. From Pfeiffer's (Note 1) model (Figure 2), it is apparent that patient protection is provided if $R_{1}, R_{2}, R_{4}$, or $R_{5}$ is made large enough, or if $R_{\mathbf{3}}$ is made small enough.

Manipulation of patient lead resistance $\left(R_{2}\right)$ and/or grounding resistance $\left(R_{1}\right)$ for patient protection may be done in several ways. Current-limiting semiconductors (usually back-to-back field effect diodes) in all patient leads $\left(R_{1}\right.$ and $\left.R_{2}\right)$ are sometimes used to update older equipment. However, the noise introduced by these components restricts their use to high-level signal applications, such as electrocardiography.

\section{Isolation Methods}

Patient input circuitry can be "floated" or electrically isolated from following circuitry $\left(R_{1}\right.$ and $R_{2}$ essentially infinity) by optical or magnetic coupling.

Magnetic isolators commonly use transformer-coupled modulation-demodulation techniques (Cromwell et al., 1973). Power to the input circuit may also be transferred at the carrier frequency to allow use of a loosely coupled, low-leakage power transformer. Cromwell et al. show a typical input circuit.

An optical isolator is essentially a light-emitting diode (LED) and photodiode combination, in which the signal information is transferred by means of a light path, eliminating the actual transfer of current across the optical barrier. The information may be transferred as an analog signal if limitations of nonlinearity and timetemperature instability are overcome. One technique uses an input-stage photodiode driven by the same LED as the output-stage photodiode to compensate for these problems. Other analog-to-digital encoding methods may be used for this purpose. Optical isolation is commonly used to couple battery-operated devices to line-powered equipment.

Whereas magnetic isolation is usually employed after the input amplifier stage, optical isolation is often used in biofeedback instruments after several stages of signal processing. The preisolator stages often use low-voltage power, usually batteries. They may also be powered by solar cells driven by an artificial light source, providing optically isolated power. These stages may also be safely powered from postisolator-stage power sources by means of an isolated dc-dc converter or from line power using an isolated power supply that meets medical standards (UL 544).

\section{Safety Grounding}

The isolation methods offer excellent protection, but the safety margin is reduced if the patient is deliberately grounded for noise-reduction purposes or makes accidental contact with grounded metal objects $\left(R_{1}\right.$ essentially zero). If power ground is accessible, the patient and operator are both in jeopardy should a power-line-to-case short occur $\left(R_{4}\right.$ equal to zero). Precautions should be taken by the user against this hazard.

A good equipment ground connection $\left(R_{3}<1\right.$ ohm $)$ is the first level of protection against this hazard situation. Integrity of this ground path is determined by checking several points. A resistance check of the ac cable ground conductor from equipment case to plug ground pin should be made while the cable is manipulated. A conductor break can occur when the ac cable is in certain positions and yet show good in other positions. Molded plugs can conceal conductor breaks; therefore, hospital-grade plugs are highly recommended. Veterans Administration hospital regulations specify that cable ground resistance should be less than $.15 \mathrm{ohm}$. Three-to-two prong ac plug adapters should not be used. Since contact resistance at the ac outlet is not a good indicator of grounding reliability (Weibell, 1974), ground pin retention force (the force required to pull the pin from the receptacle) should also be tested. It should be determined that Terminal $\mathrm{C}$ at the receptacle (Figure 3 ) is connected to earth ground by a large copper conductor, not by means of conduit pipe. It should also be confirmed that the hot terminal and either neutral or ground have not been transposed at the receptacle or in the ac cable.

\section{Ground-Fault Circuit Interrupter}

As seen earlier, even a good ground connection may not be sufficient protection against a direct ground fault. A device known as a ground-fault circuit interrupter (GFCI) interrupts power quickly (e.g., $25 \mathrm{msec}$ ) in case of a ground fault, giving a second level of protection. A GFCI will also interrupt power in the event that someone's body provides a path from an energized ungrounded chassis to ground in which a flow of more than 4 to $6 \mathrm{~mA}$ results. The GFCI compares the current in the hot side of the line to that in the neutral side. An unintended path to ground causes an imbalance between these currents. If the difference exceeds about $5 \mathrm{~mA}$, the GFCI interrupts power. A GFCI does not protect against (unlikely) simultaneous contact with the hot and neutral sides of the line. While the GFCI affords a measure of protection for both operator and patient, the device is sensitive to imbalances due to leakage currents and may be tripped by equipment with RF line filters, motors, or other leakage sources. Such equipment is not likely to meet the AAMI standard for ground conductor risk current. A GFCI can readily be added to existing facilities in the form of a replacement circuit breaker, a replacement wall outlet, or a unit that plugs 
directly into the existing outlet. They range in price from $\$ 30$ to $\$ 50$ from most electrical supply houses.

\section{Insulation}

Double insulation can provide an additional safeguard against hazards involving the equipment case for both patient and operator, by protective insulation around conductive equipment surfaces in addition to the primary functional insulation. Devices that are completely battery powered may have metal cabinets that should be isolated from the conductive surfaces of line-powered equipment.

\section{Microshock Precautions}

The foregoing safety measures should provide adequate protection for both patient and operator in most current biofeedback applications. Use of instruments with electrically susceptible patients, however, requires additional precautions. The patient isolation methods, combined with an equipotential grounding system, would provide a high level of protection. Equipotential grounding establishes a single patient grounding point to avoid currents due to differences in ground potential in the patient's environment. Lowresistance (less than $.1 \mathrm{ohm}$ ) paths connect all receptacle grounds and accessible conductive surfaces in a patient's vicinity.

Isolated power-distribution systems utilizing isolation transformers are generally considered too expensive for the protection they provide. These systems attempt to establish an extremely high value of line ground resistance, $R_{5}$, by not grounding the isolation transformer secondary. A line isolation monitor or groundfault detector monitors the leakage current from each side of the secondary to ground. This device, like the GFCI, may be tripped by equipment leakage, interrupting the power to the system. The line ground resistance should be maintained so as to be essentially zero in most power-distribution systems.

\section{SUMMARY}

The user of biofeedback instruments is fortunate to be working in an area served by manufacturers who have demonstrated a willingness to self-regulate and use the latest techniques to assure patient safety. However, the user must share the responsibility for electrical safety. Safety recommendations to the user can be summarized as follows.
(1) The user should be aware of the safety measures built into a commercial device by the manufacturer and not attempt to defeat them. If, for example, a battery-powered instrument is to be connected to a line-powered device, it should be determined that protection is still provided.

(2) The integrity of the equipment grounding path of line-powered equipment should be checked regularly as outlined under Safety Grounding. The Veterans Administration requires a quarterly inspection of equipment grounding resistance. The Veterans Administration also requires annual inspection of ac wall receptacles for polarity (hot and neutral), ground conductor integrity, contact tension, and overall physical condition.

(3) A ground-fault circuit interrupter is highly recommended for back-up protection with line-powered equipment.

(4) Appliances such as lamps and radios and grounded surfaces should be kept well out of the reach of the patient or anyone contacting the patient.

(5) Electrically susceptible patients require special protective measures, such as equipotential grounding systems and isolated circuitry. Microshock precautions, of course, provide protection against macroshock.

Any safety measure can fail. Therefore, the key to electrical safety is to provide back-up protection in the event of breakdown or malfunction.

\section{REFERENCE NOTE}

1. Pfeiffer, E. A. Electrical safety in the hospital. 1972 WESCON Technical Papers 12/3.

\section{REFERENCES}

BerNstein, T. Electrical safety in aversive conditioning of humans. Behavioral Engineering, 1975, 2, 31-34.

Cromwell, L., Weibell, F. J., Pfeiffer, E. A., \& Usselman, L. B. Biomedical instrumentation and measurements. Englewood Cliffs, N.J: Prentice-Hall, 1973.

DALzIEL, C. F. Electric shock hazard. Institute of Electrical and Electronics Engineers Spectrum, 1972, 9, 41-50.

Pfeiffer, E. A. Electrical stimulation of sensory nerves with skin electrodes for research, diagnosis, communication and behavioral conditioning: A survey. Medical and Biological Engineering, 1968, 6, 637-651.

Pope, A. T., \& Gersten, C. D. Computer automation of biofeedback training. Behavior Research Methods \& Instrumentation, 1977, 9, 164-168.

WEIBELl, F. J. Electrical safety in the hospital-1974. Annals of Biomedical Engineering, 1974, 2, 126-148. 